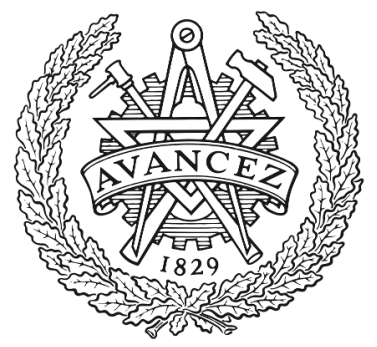

CHALMERS

UNIVERSITY OF TECHNOLOGY

\title{
Clustering and Morphology Evolution of Gold on Nanostructured Surfaces of Silicon Carbide: Implications for Catalysis and Sensing
}

Downloaded from: https://research.chalmers.se, 2023-04-26 09:58 UTC

Citation for the original published paper (version of record):

Shtepliuk, I., Ivanov, I., Pliatsikas, N. et al (2021). Clustering and Morphology Evolution of Gold on Nanostructured Surfaces of Silicon Carbide:

Implications for Catalysis and Sensing. ACS Applied Nano Materials, 4(2): 1282-1293.

http://dx.doi.org/10.1021/acsanm.0c02867

N.B. When citing this work, cite the original published paper. 


\title{
Clustering and Morphology Evolution of Gold on Nanostructured Surfaces of Silicon Carbide: Implications for Catalysis and Sensing
}

\author{
Ivan Shtepliuk,* Ivan G. Ivanov, Nikolaos Pliatsikas, Tihomir Iakimov, Samuel Lara-Avila, \\ Kyung Ho Kim, Nabiha Ben Sedrine, Sergey E. Kubatkin, Kostas Sarakinos, and Rositsa Yakimova
}

Cite This: https://dx.doi.org/10.1021/acsanm.0c02867

Read Online

ACCESS I

Џll Metrics \& More

Article Recommendations

Supporting Information

ABSTRACT: A fundamental understanding of the behavior of gold $(\mathrm{Au})$ nanostructures deposited on functional surfaces is imperative to discover and leverage interface-related phenomena that can boost the efficiency of existing electronic devices in sensorics, catalysis, and spintronics. In the present work, $\mathrm{Au}$ layers with nominal thickness of $2 \mathrm{~nm}$ were sputter-deposited on graphenized $\mathrm{SiC}$ substrates represented by buffer layer $(\mathrm{BuL}) / 4 \mathrm{H}-\mathrm{SiC}$ and monolayer epitaxial graphene (MLG)/4H-SiC. Morphometric analysis by means of scanning electron microscopy shows that Au on BuL self-assembles in nearly round-shaped plasmonically active islands, while on MLG, a fractal growth of considerably larger and ramified islands is observed. To correlate

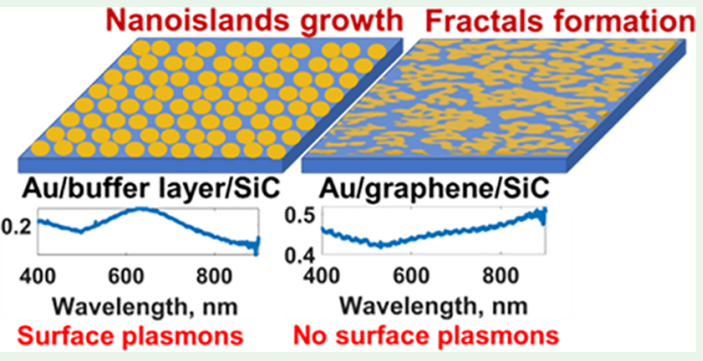
the experimentally established differences in surface morphology on the two types of graphenized substrates with energetics and kinetics of $\mathrm{Au}$ nanostructure growth, the deposit-substrate interaction strength was studied using density functional theory (DFT) calculations, molecular dynamics simulations, and optical measurements. The theoretical considerations involve participation of Au clusters with different sizes and energetics at the initial stages of the metal nanostructure formation. The results indicate that gold exhibits a considerably stronger interaction with BuL than with MLG, which can be considered as a key aspect for explaining the experimentally observed morphological differences. From the statistical analysis of Raman spectra, indications of Au intercalation of MLG are discussed. The current research shows that, due to its unique surface chemistry, buffer layer has peculiar affinity to gold when compared to other atomically flat surfaces, which is beneficial for boosting high-performance catalytic and sensing technologies based on low-dimensional materials.

KEYWORDS: gold, epitaxial graphene, SiC, buffer layer, adsorption, growth

\section{INTRODUCTION}

Monolayer epitaxial graphene on the Si face of $4 \mathrm{H}-\mathrm{SiC}$ (MLG/ $\mathrm{SiC}$ ) is an important ingredient of today's advanced technologies in quantum metrology, ${ }^{1-3}$ electronics, ${ }^{4-6}$ and sensorics. $^{7-10}$ Due to several fundamental and practical advantages over other graphene-family materials, ${ }^{11} \mathrm{MLG} / \mathrm{SiC}$ has gained wide-ranging recognition as a multifunctional monolithic architecture, which can perfectly satisfy application-specific requirements while maintaining the key properties of true graphene crystal. An expected step in the development of epitaxial graphene devices is the integration of $\mathrm{MLG} / \mathrm{SiC}$ with noble metals (NMs) to complement or expand the functionalities of the existing $\mathrm{MLG} / \mathrm{SiC}$ host system. For instance, interfacing gold $(\mathrm{Au})$ with epitaxial graphene has a huge potential to be exploited as (i) ohmic contact, due to a low Au-MLG/SiC contact resistivity of $<1 \times 10^{-6} \Omega \cdot \mathrm{cm}^{2},{ }^{12}$ (ii) a catalyst for electrochemical hydrogenation of $M L G,{ }^{13}$ (iii) an active sensing component on MLG for detection of biomolecules and gases, ${ }^{14-16}$ and (iv) a plasmonically active phase enabling SERS (surface-enhanced Raman scattering) applications. ${ }^{17,18}$ In this regard, atomistic-level interfacial processes governing the growth energetics and kinetics of $\mathrm{Au}$ and other NM nanostructures and layers on epitaxial graphene have attracted considerable attention during the last years. ${ }^{19-23}$ Indeed, critical knowledge of the mutual interplay between NMs and MLG/SiC is required to accelerate the development of tunable NM-MLG/SiC hybrids and related devices.

In addition to MLG growth, another unique opportunity provided by $\mathrm{SiC}$ is the growth of the so-called buffer layer $(\mathrm{BuL})$, formed at the early stages of the annealing process used for MLG synthesis. The $\mathrm{BuL}$ is a $\mathrm{C}$-rich reconstructed $\mathrm{SiC}$ surface, which is partly bonded to the substrate and can be decoupled from the substrate by elemental intercalation and transformed to quasi-free standing (QFS) monolayer graphene. Some of the technological implications of buffer layer have been described in our previous works, ${ }^{24,25}$ where BuL in place of MLG enables homogeneous deposition of metals. The

Received: October 26, 2020

Accepted: January 7, 2021 
homogeneous deposition of $\mathrm{Pt}$ is only possible on buffer layers $^{25}$ and not on MLG (nor other atomically flat surfaces such as $4 \mathrm{H}-\mathrm{SiC}$ or sapphire). In ref 24 , we describe that buffer layer allows the formation of atomically thin $\mathrm{Pt}$, which works as a chemical sensor with sub-part-per-billion sensitivities and fast operation. In this regard, buffer layer, which has been thought as a technological barrier for epitaxial graphene technology due to high intrinsic doping, is now considered to have its own technological relevance for catalysis and biosensing. Therefore, its high affinity to transition metals needs to be studied comprehensively in comparison with MLG surfaces. However, the major focus of existing studies of the $\mathrm{Au}-\mathrm{BuL}$ system is the controllable $\mathrm{Au}$ intercalation beneath $\mathrm{BuL}$ to form high-quality QFS graphene with tunable properties. ${ }^{26-34}$ For instance, homogeneous deposition of $\mathrm{Au}$ on BuL followed by annealinginduced $\mathrm{Au}$ intercalation enables ambipolar charge transport of quasi-freestanding graphene, ${ }^{25}$ which may find applications in sensorics and spin-related transport. Au as intercalant species is also anticipated to initiate new physical phenomena at the interface, including tuning the energy scale of van Hove singularity and Fermi velocity increase, ${ }^{34}$ doping control, ${ }^{31}$ and giant Rashba-type spin-orbit splitting. ${ }^{28}$ Although substantial progress has been made towards clarifying $\mathrm{Au}$ intercalation conditions and identification of Au intercalants' morphology, the resulting interplay between graphene and the confined gold atoms after intercalation is still an open issue. There are hints that the morphology of intercalated $\mathrm{Au}$ atoms determines the properties of the resulting graphene-Au intercalated systems. ${ }^{25,26,35}$

Based on the analysis of the existing scientific background and applications, it can be stated that the interaction between gold and graphenized $\mathrm{SiC}$ is decisive for the applicationspecific properties of the Au-MLG and Au-BuL systems. Fine tuning this interaction can enable manipulating Au morphology from dispersed three-dimensional (3D) nanoscale islands to continuous two-dimensional (2D) layers. Thus, a holistic understanding of $\mathrm{Au}$ adsorption and kinetics on the graphenized $\mathrm{Si}$ face of on-axis $4 \mathrm{H}-\mathrm{SiC}$ is an essential precondition to realize a full control on the structural morphology of deposited and/or intercalated Au. Furthermore, because of the complex structure of the $\mathrm{MLG} / \mathrm{BuL} / \mathrm{SiC}$ system that offers several options for $\mathrm{Au}$ accommodation, it is of fundamental importance to distinguish between the processes occurring on the buffer layer and those on the graphene monolayer. Despite the pronounced interest in the investigation of Au behavior on graphenized $\mathrm{SiC}$, such distinction is still not fully explored and remains a challenging task for researchers.

In this work, we shed light on fundamental aspects of $\mathrm{Au}$ nanostructures grown by direct current (d.c.) magnetron sputtering on buffer layer and monolayer epitaxial graphene synthesized on the $\mathrm{Si}$ face of on-axis $4 \mathrm{H}-\mathrm{SiC}$ by the wellrecognized method of high-temperature thermal decomposition in argon atmosphere. ${ }^{36}$ As far as the authors are aware, there are no studies reported on selective gold growth on $\mathrm{BuL}$ with MLG patches by d.c. magnetron sputtering. Considering the high technological importance of the magnetron sputtering for the formation of homogeneous metallic coatings on largearea substrates, it is sensible to investigate the morphology of vapor-deposited gold layers on both BuL and MLG and to link the structural uniqueness of gold on each type of surfaces to the specific gold-template interaction, which is a key determining factor in processes of nucleation, diffusion, and clustering. By performing complementary experimental work and $\mathrm{ab}$ initio calculations, we first demonstrate fundamentally important material-property relationships for the Au-graphenized $\mathrm{SiC}$ system. We are focusing on the investigation of the mesoscopic morphology of gold on graphenized $\mathrm{SiC}$ rather than on the investigation of detailed morphology features of the $\mathrm{Au}$ nanoclusters. We show that the $\mathrm{BuL}$ acts as a strongly interacting substrate for gold deposition and the presence of $\mathrm{sp}^{3}$-bonded $\mathrm{C}$ atoms limits the diffusion length of $\mathrm{Au}$, which results in the formation of high-density isolated round islands. Concomitantly, we observe growth of larger and ramified irregularly shaped $\mathrm{Au}$ islands on MLG, which is explained considering the weak interaction between gold and epitaxial graphene and strong metal-metal attractive forces. The obtained results provide yet-unknown in-depth atomistic and thermodynamic insights into the nature of the interaction between gold and BuL or MLG, thereby expanding the existing knowledge on gold self-assembling on atomically thin, flat, and corrugated substrates as well as promoting further development of the mentioned system for biosensing and catalysis.

\section{METHODS}

2.1. Experimental Procedures. To distinguish between the behavior of $\mathrm{Au}$ on buffer layer and graphene monolayer, we prepared corresponding templates by high-temperature $\left(\sim 1700\right.$ to $\left.1900{ }^{\circ} \mathrm{C}\right)$ thermal decomposition of the $\mathrm{Si}$ face of nominally on-axis $4 \mathrm{H}-\mathrm{SiC}$ (0001) substrates in Ar atmosphere. ${ }^{36}$ The graphenization proceeds via $\mathrm{Si}$ sublimation in an inductively heated graphite enclosure under strictly controlled temperature-gas pressure-time conditions, enabling to achieve a complete coverage of the $4 \mathrm{H}-\mathrm{SiC}$ substrate with the C-rich $6 \sqrt{3} \times 6 \sqrt{3} \mathrm{R} 30^{\circ}$ surface pattern $(\mathrm{BuL})$ due to characteristic reconstruction of the (0001) face, also known as zero graphene layer. While heating, BuL transforms into monolayer epitaxial graphene (MLG) above a subsequent buffer layer. Therefore, we prepared two types of graphenized surfaces: buffer layer and monolayer graphene over a buffer layer, respectively. BuL formation was realized at a lower temperature in comparison to the MLG growth temperature, and the $\mathrm{BuL}$ templates intentionally contain MLG graphene inclusions that give the opportunity to compare the different surface properties of $\mathrm{BuL}$ and MLG on the same $\mathrm{SiC}$ substrate. Both studied templates have atomic thicknesses and twodimensional structures providing nonstandard substrates for further nanoscale metal deposition and related phenomena. Prior to the $\mathrm{Au}$ deposition process, we performed detailed Raman and optical reflectance mappings of the substrates to evaluate the quality of the carbon layers (thickness, homogeneity, defects, etc.). The Raman fingerprint of $\mathrm{BuL}$, which includes structured bands within the frequency range of $1355-1600 \mathrm{~cm}^{-1,37}$ was observed at every set point of the investigated area, suggesting a homogeneous $\mathrm{BuL}$ coverage (see Figure S1, Supporting Information), typically with few submicrometer patches of MLG. The formation of MLG was then evidenced by the observation of the characteristic $G$ peak (around $1602.78 \pm 3.55 \mathrm{~cm}^{-1}$ ) and $2 D$ peak (around $2738.73 \pm 6.42 \mathrm{~cm}^{-1}$ ) during Raman mapping (Figure S2, Supporting Information). This observation is consistent with the topmost graphene layer being $\mathrm{n}$ doped and under biaxial compressive strain. ${ }^{38}$ Optical reflectance mapping (Figure S3, Supporting Information) confirmed that most of the $\mathrm{SiC}$ surface $(\sim 81 \%)$ is covered by monolayer graphene, while the remaining $\sim 19 \%$ are small inclusions of bilayer epitaxial graphene (BLG) regions. Notably, no defect-related $D$ phonon mode was observed in the Raman spectra. This indicates a high crystalline quality of the epitaxial graphene, required to avoid defect-mediated $\mathrm{Au}$ nucleation that might mask eventual van der Waals epitaxy of $\mathrm{Au}$ on the basal plane of graphene.

Au layers with a nominal thickness of $2 \mathrm{~nm}$ were deposited on $\mathrm{BuL}$ and MLG by direct current (d.c.) magnetron sputtering of an $\mathrm{Au}$ target at room temperature and UHV conditions (base pressure < 
Scheme 1. Illustrative Summary of the Experimental Methodology Used for Synthesis of Au-BuL and Au-MLG Samples

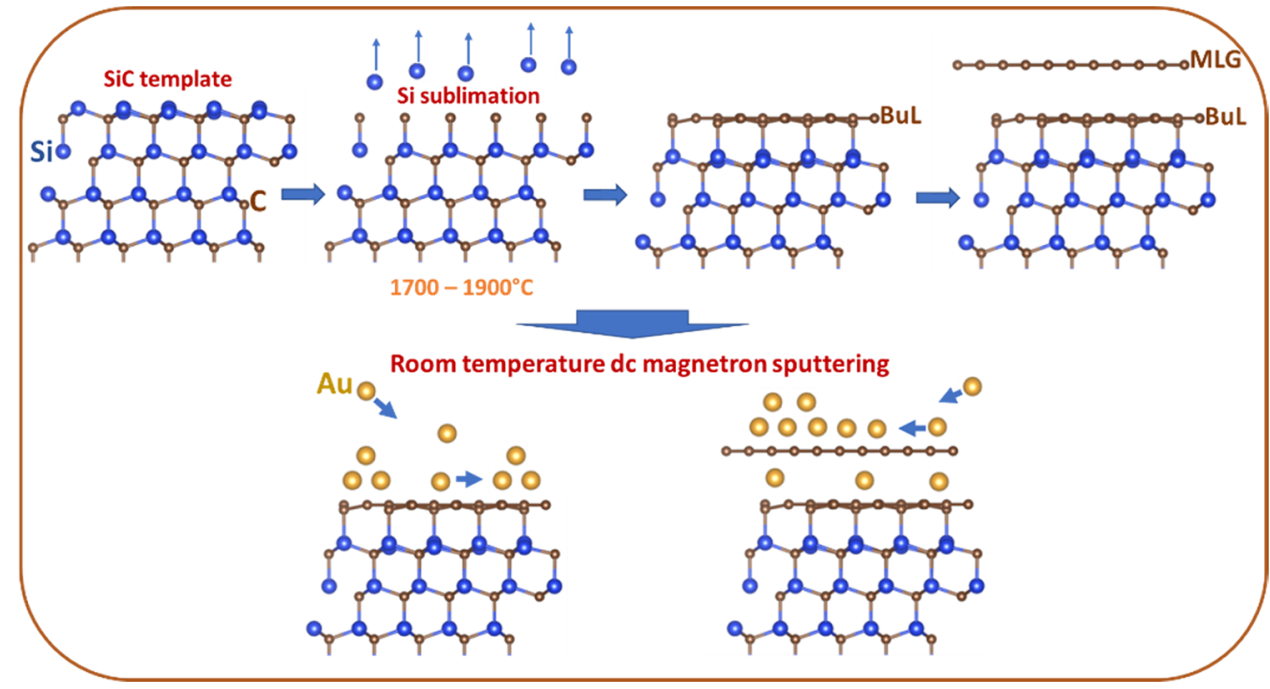

$10^{-8} \mathrm{~Pa}$ ). The total pressure of $\mathrm{Ar}$ (purity 99.999\%) in the working chamber was kept at $6.7 \mathrm{~Pa}$ (50 mTorr), while the deposition rate was maintained at $0.1 \AA / \mathrm{s}$. Such mild deposition conditions were used to ensure that the epitaxial graphene remains undamaged after magnetron sputtering of Au. From our previous work, with the growth of noble metals on weakly interacting substrates, ${ }^{19,20,24,25,39}$ a $2 \mathrm{~nm}$ thickness results in a discontinuous metal layer that allows us to study film morphology while leaving a part of graphene exposed for various analyses. Concurrently, differences in morphology observed during the stage of discontinuous layer translate consistently into differences in morphology of continuous layers (i.e., more pronounced 3D morphology of islands in discontinuous layer typically means larger roughness and a continuous film is formed). Scheme 1 summarizes the steps used in the preparation of the gold-decorated $\mathrm{BuL}$ and MLG samples, respectively. To explore whether the observed gold morphologies are related with features of the sputtering process (energetic bombardment, plasma, etc.) or they are processindependent and can only be ascribed to the film/substrate system, extremely thin gold nanofilms were also deposited using thermal evaporation and additionally studied by atomic force microscopy (AFM).

The surface morphology and microstructure of the as-deposited $\mathrm{Au}$ films were examined by scanning electron microscopy (SEM) (Leo 1550 Gemini SEM instrument) at an operating voltage ranging from 10 to $20 \mathrm{kV}$ and a standard aperture value of $30 \mathrm{~mm}$. The quality of the Au-coated graphenized surfaces and the effect of the Au deposits on the phonon modes of graphene and BuL were explored by microRaman spectroscopy mapping. Raman spectra were recorded with a micro-Raman setup based on a monochromator (Jobin-Yvon, model HR460) equipped with a CCD (couple-charged device) camera. The objective lens has a magnification of $100 \times$ and numerical aperture (NA $=0.95)$ resulting in an $\sim 0.85 \mu \mathrm{m}$ diameter of the laser spot focused on the sample surface. A $532 \mathrm{~nm}$ diode-pumped solid-state laser with $1 \mathrm{~mW}$ power was used as an excitation source. The spectral resolution of the system is $\sim 5.5 \mathrm{~cm}^{-1}$. Absorbance measurements were performed at room temperature using a Shimadzu UV-2100 spectrometer.

2.2. Theoretical Details. To elucidate the specifics of Au growth kinetics and energetics, we studied theoretically $\mathrm{Au}$ behavior on two different interfaces, namely, $\mathrm{BuL} / 4 \mathrm{H}-\mathrm{SiC}$ and $\mathrm{MLG} / \mathrm{BuL} / 4 \mathrm{H}-\mathrm{SiC}$. We employed the $\sqrt{3} \times \sqrt{3} \mathrm{R} 30^{\circ}$-reconstructed $4 \mathrm{H}-\mathrm{SiC}(0001)$ substrate (Figure S4), which is consistent with the experimentally observed features of the MLG/BuL/4H-SiC system. ${ }^{40,41}$ According to this model, a $2 \times 2$ honeycomb array of carbon atoms is accommodated on top of the reconstructed surface of $4 \mathrm{H}-\mathrm{SiC}$ (0001). To satisfy charge neutrality conditions, the dangling bonds of the bottom carbon layer (or carbon-terminated face) of a $4 \mathrm{H}-\mathrm{SiC}$ slab were passivated by forming $\mathrm{C}-\mathrm{H}$ bonds. The buffer layer is partly covalently bonded to the $\mathrm{Si}$ surface atoms of the $\mathrm{SiC}$ and is known to be corrugated. It does not exhibit graphene-like properties, while the first graphene layer above the $\mathrm{BuL}$ is an $\mathrm{n}$-doped honeycomb array of $\mathrm{C}$ atoms with a cone-like electronic band structure.

To study the $\mathrm{Au}$ atom adsorption and migration on the graphenized $\mathrm{SiC}$ surfaces, we used the following scheme: (1) positioning the $\mathrm{Au}$ atom at the specific site (top site) in the beginning of the migration path $\rightarrow$ (2) geometrical optimization of the Cartesian coordinates with constraints to Au movements in $x$ and $y$ directions at the starting point of the diffusion path $\rightarrow(3)$ physical movement of the $\mathrm{Au}$ atom to the next point along the migration path followed by relaxation only along the $z$ direction (Figure S5, Supporting Information). Despite the fact that thermal vibrations may modify diffusion behavior, the static potential energy landscapes calculated from energy minimization can provide a qualitative meaningful picture of the adsorption and migration dynamics in weakly interacting film/substrate systems, which is consistent with experimental results. ${ }^{42-45}$ The calculated adsorption energy difference at specific sites determines how long an adsorbed $\mathrm{Au}$ atom will remain trapped at the surface of interest: the larger the adsorption energy difference, the shorter the diffusion path. ${ }^{46}$ We also studied the $\mathrm{Au}$ nucleation process on the considered interfaces by using larger slabs: $4 \times 4 \mathrm{BuL} / 4 \mathrm{H}-\mathrm{SiC}$ and $4 \times 4 \mathrm{MLG} / \mathrm{BuL} / 4 \mathrm{H}-\mathrm{SiC}$, respectively (see Figure S6, Supporting Information).

All $a b$ initio calculations were carried out by using the SIESTA $\operatorname{code}^{47}$ within the van der Waals density functional (vdW-DF) method. ${ }^{48}$ The construction of pseudopotentials for $\mathrm{H}, \mathrm{Si}, \mathrm{C}$, and $\mathrm{Au}$ was performed within the Troullier-Martins scheme by using the ATOM code. ${ }^{49}$ A vacuum layer of $20 \AA$ was added above the graphenized surface along the slab-normal direction to avoid undesired interactions with neighboring unit cells. Double- $\zeta$ polarized (DZP) basis set with an energy shift of $200 \mathrm{meV}$ was chosen. Although DZP basis set usually allows obtaining reasonable results, some extra calculations using larger basis sets have been carried out to validate the results of the model (see Section S1, Supporting Information). The Cartesian coordinates of the considered structures were relaxed until the force on each atom reached less than $0.02 \mathrm{eV} /$ A. A Monkhorst-Pack $k$-point $3 \times 3 \times 1$ mesh was used to sample the Brillouin zone during the optimization process. The mesh cutoff was set at $400 \mathrm{Ry}$. All obtained results are strictly valid only at $T=0 \mathrm{~K}$ and provide deeper insights into the nature of the low-energy properties of $\mathrm{Au}$-template systems. A better understanding of ground-state properties will benefit further experimental work and future applications, since fundamentally crucial material-property relationships can be extrapolated to temperatures beyond $0 \mathrm{~K}$. 

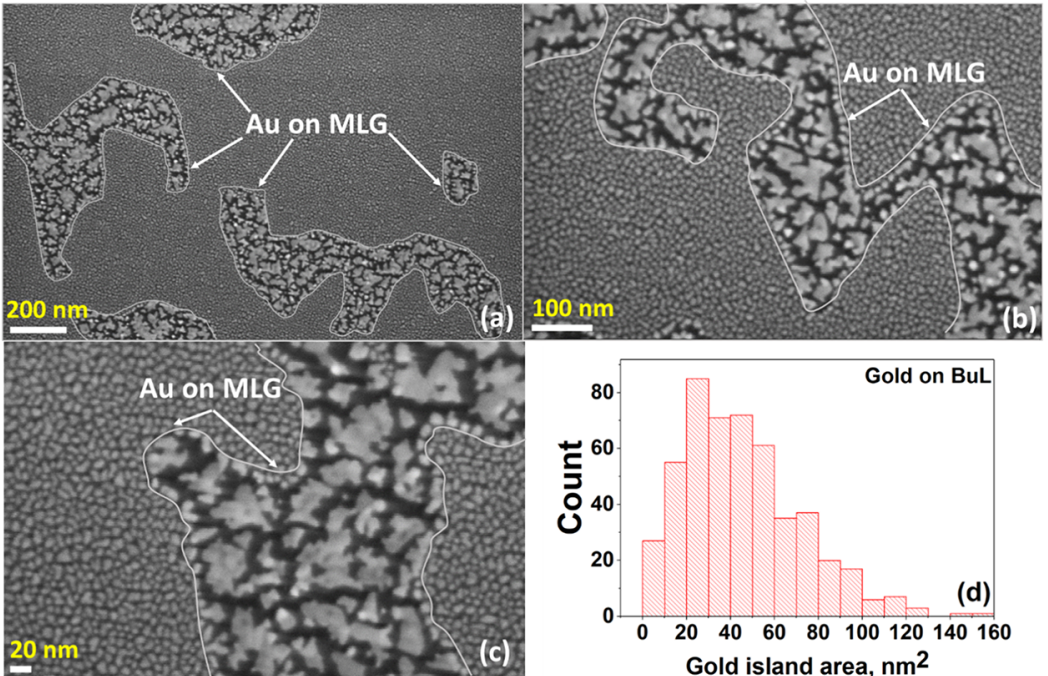

Figure 1. $(a-c)$ SEM images of Au islands grown on the buffer layer. Small patches of MLG are observed, which lead to distinctly different morphologies relative to the surrounding area and further highlights the effect of the underlying surface on Au nanostructure growth. (d) Particle size distribution histogram derived from SEM images of $\mathrm{Au}$ on $\mathrm{BuL}$.

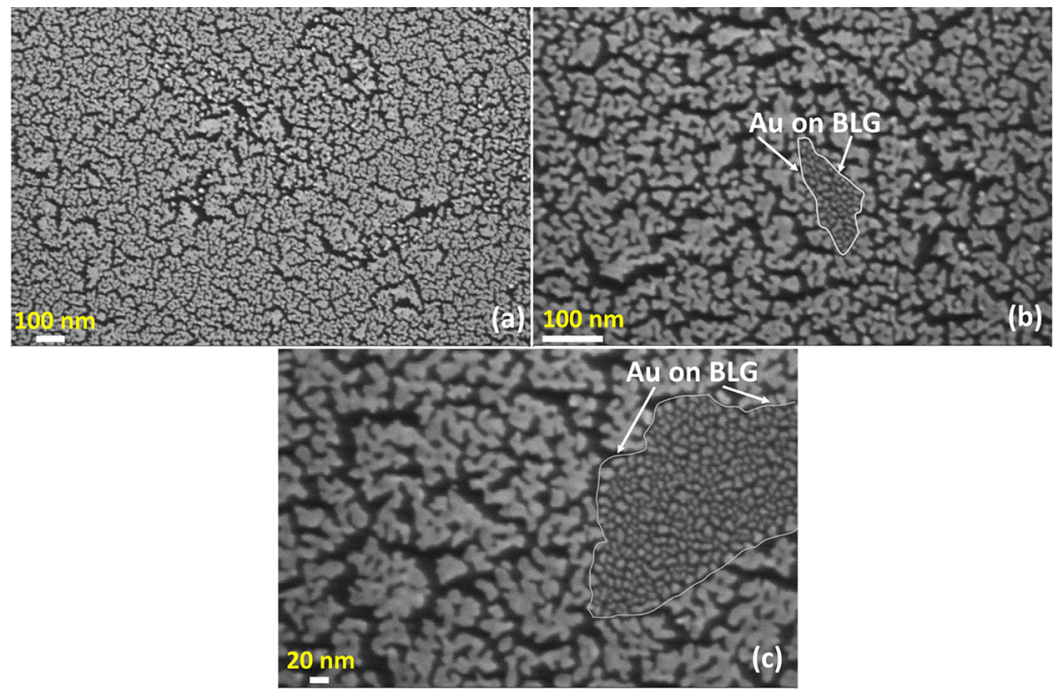

Figure 2. (a-c) SEM micrographs of the Au deposited on MLG. A small patch with Au morphology resembling that on BuL is observed near the center of this image. Since the reflectance map shows only MLG and bilayer coverage, we can ascribe this patch to the gold layer formed on bilayer epitaxial graphene (BLG). Gold on BLG assembles in smaller and denser elongated islands due to expected larger adsorption energy of Au on bilayer graphene compared to that on monolayer graphene. However, existence of submicrometer patches of BuL on this sample cannot be precluded because their appearance within the laser spot $(\sim 0.85 \mu \mathrm{m}$ in diameter) would have negligible impact on the reflectance.

\section{RESULTS AND DISCUSSION}

\subsection{Morphology of Au Deposited on Buffer Layer} and Monolayer Epitaxial Graphene. As a first step toward understanding energetic and kinetic aspects of $\mathrm{Au}$ growth on graphenized surfaces of $4 \mathrm{H}-\mathrm{SiC}$, we studied experimentally the morphology of as-deposited layers on both buffer layer and monolayer epitaxial graphene using SEM. In the case of BuL/ $\mathrm{SiC}$ substrates (Figure $1 \mathrm{a}-\mathrm{c}$ ), Au self-assembles into largely isolated and nearly circular nanoscale islands with no evident interconnections between them. Analysis of the SEM images shows that the island density is $9.6 \times 10^{11} \mathrm{~cm}^{-2}$, while the substrate area coverage is $43.7 \%$. Moreover, the island size (i.e., projected area) follows a bell-shaped distribution with a mean size of $45.6 \mathrm{~nm}^{2}$ (Figure 1d). We also note that some parts of the BuL substrate contain MLG overlaid regions (see the outlined regions in Figure $1 \mathrm{a}-\mathrm{c}$ ), which show completely different $\mathrm{Au}$ morphology, i.e., $\mathrm{Au}$ islands are considerably larger in size and exhibit dendritic shapes.

The overgrowth of monolayer graphene layers on $\mathrm{BuL}$ allows to study the Au growth simultaneously on both surfaces and to elucidate the difference in Au growth morphology on $\mathrm{BuL}$ and MLG. The differences in growth morphology on the two types of graphenized surfaces are further confirmed by the SEM data from the $\mathrm{Au} / \mathrm{MLG} / \mathrm{SiC}$ sample (Figure 2), which reveal the formation of islands with an approximate mean size of $1623.7 \mathrm{~nm}^{2}$ and ramified island shapes (note that the island size in Figure 2 was estimated only based on the analysis of the isolated islands, while most of the MLG surface is covered with large and dendritic $\mathrm{Au}$ structures).

A similar picture was observed for $4 \AA$ gold deposited by thermal evaporation on BuL with MLG patches (see Figure 3). In this case, $\mathrm{Au}$ completely covers the BuL forming a smooth 

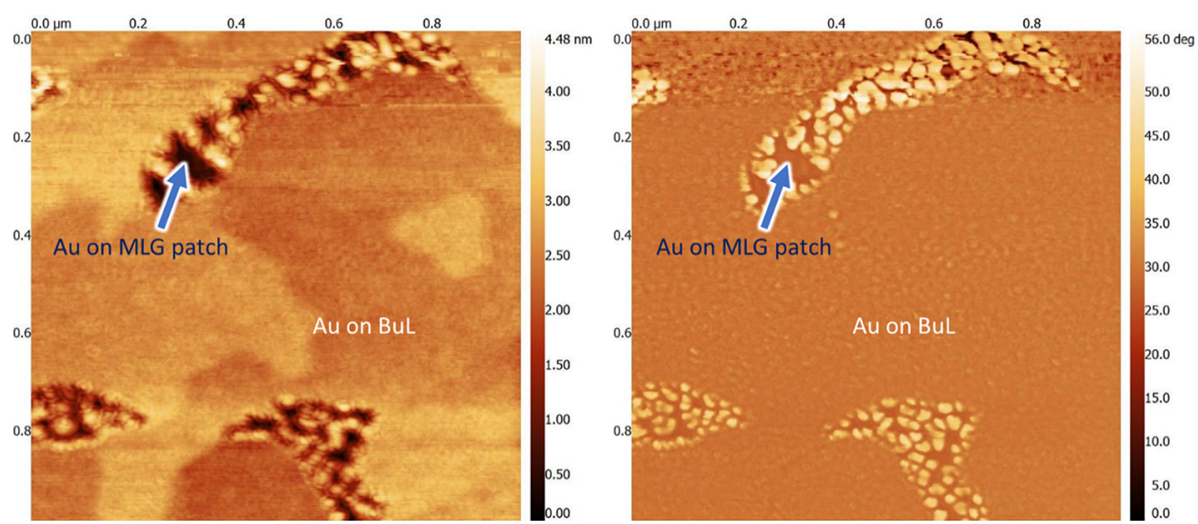

Figure 3. (Left panel) AFM image and (right panel) phase image of $4 \AA$ A-thick Au deposited onto the $\mathrm{BuL} / 4 \mathrm{H}-\mathrm{SiC}$ surface by the thermal evaporation technique, respectively. Field of view is $1 \mu \mathrm{m} \times 1 \mu \mathrm{m}$, respectively.

coverage. On the other hand, a pronounced Au agglomeration takes place on the MLG patches (see regions indicated by the arrows in Figure 3). The morphological similarities of $\mathrm{Au}$ layers obtained by different deposition methods illustrate a common trend in $\mathrm{Au}$ growth on graphenized $\mathrm{SiC}$ surfaces. More details on the specific structural features of thermally evaporated gold layers on graphenized surfaces of $\mathrm{SiC}$ studied by using scanning tunneling microscopy (STM) and synchrotron facilities can be found in our recently published work, $^{25}$

The analysis of the SEM image of sputter-deposited Au on MLG (Figure 2) shows that the substrate coverage is $36.6 \%$, this being smaller than that for the $\mathrm{Au} / \mathrm{BuL} / \mathrm{SiC}$ system (43.7\%). Given that on both surfaces the same amount of $\mathrm{Au}$ was deposited, we conclude that Au exhibits more pronounced 3D growth morphology on MLG compared to BuL. From a thermodynamic point of view, the observed results could be understood by considering the difference between the surface energies of the two substrates. Since the surface energy is strongly dependent on the structural parameters (including geometric configuration of the adsorption sites) and electronic properties of the substrate, it positively correlates with the reactivity of the surface sites and, consequently, with the adsorption energy of external adsorbates: ${ }^{50}$ the larger the surface energy, the higher the adsorption energy. In our case, the surface energy of MLG $\left(0.1 \mathrm{~J} / \mathrm{m}^{2}\right)^{51}$ is much smaller compared to that of $\mathrm{BuL}$, thereby providing lower wettability and weaker nanoscale adsorption of $\mathrm{Au}$ during early growth stages. $^{52}$ It should be noted, however, that the surface energy of $\mathrm{BuL}$ is not known exactly and is assumed to be close to that of the nongraphenized $\mathrm{SiC}$ substrate $\left(1.4 \mathrm{~J} / \mathrm{m}^{2}\right) .{ }^{53}$ Because of the substantial difference in the surface energetics of the two material systems, it seems that the morphology of $\mathrm{Au}$ deposited on MLG is insensitive ${ }^{54}$ to the underlying buffer layer i.e., the contribution of $\mathrm{Au}-\mathrm{BuL}$ interaction to the total Au-MLG/BuL interaction is negligibly small, which can be probably related to prevailing van der Waals forces on MLG. By using ImageJ software with the FracLac plugin, we performed additional analysis of the dendritic microstructure of $\mathrm{Au}$ islands on MLG and found that the fractal dimension is approximately equal to 1.84 . Due to the thermalized nature of the deposition fluxes used in our experiments (sputtering at 50 mTorr) that cause limited atomic diffusion at island edges, the diffusion-limited aggregation (LDA) is believed to be a key mechanism underlying the formation of dendritic structures exhibiting fractal dimensions. ${ }^{55}$ According to this mechanism, the surface diffusion of the as-deposited gold atom can be considered as the random walk on the weakly interacting substrate containing a preformed stable nucleus. Once this gold atom reaches the nucleus, its further movement is constrained. Newly arriving gold species repeat this process, thereby forming the fractal aggregates.

Finally, we noticed that the observed morphological properties of gold correlate with its optical properties (Figure 4). More specifically, dendritic gold nanostructures on MLG

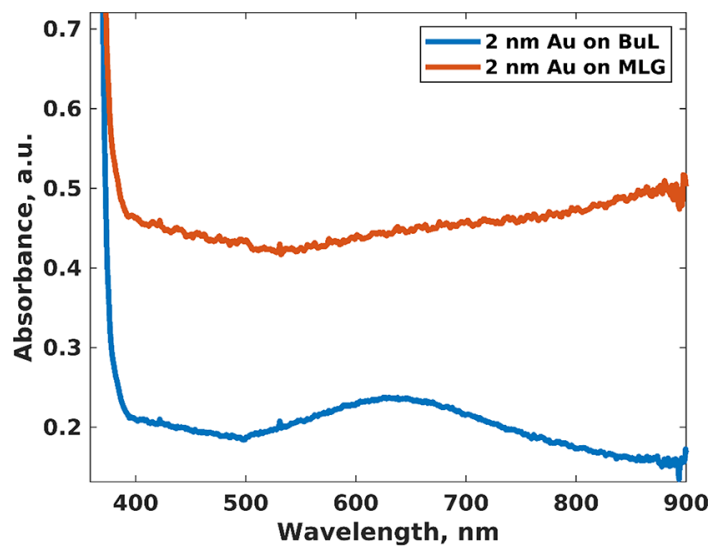

Figure 4. Absorption spectra of $2 \mathrm{~nm}$ gold deposited on the buffer layer (blue curve) and monolayer (orange curve), respectively, as measured with UV-visible spectroscopy.

show a higher absorbance compared to gold on BuL, without any pronounced feature. Meanwhile, isolated $\mathrm{Au}$ nanoscale islands on BuL exhibit an absorption band at $634 \mathrm{~nm}$ related to localized surface plasmon resonance. This feature can be used for design of highly selective sensor arrays based on buffer layer for optical detection of biomolecules.

To understand the way by which morphology differences are related with the fundamental processes of adatom adsorption and diffusion, we study the deposit/substrate interactions using Raman spectroscopy and DFT calculations, as presented in the next two sections.

3.2. Probing Gold-Substrate Interaction by Raman Spectroscopy. Figure 5 displays typical Raman spectra taken from $\mathrm{Au} / \mathrm{MLG} / \mathrm{BuL} / 4 \mathrm{H}-\mathrm{SiC}, \mathrm{Au} / \mathrm{BuL} / 4 \mathrm{H}-\mathrm{SiC}$, and the corresponding reference samples (MLG/BuL/4H-SiC and $\mathrm{BuL} / 4 \mathrm{H}-\mathrm{SiC}$ ). As seen from this figure (cf. also Figure $\mathrm{S} 1$ in the Supporting Information), the Raman spectrum of the bare 


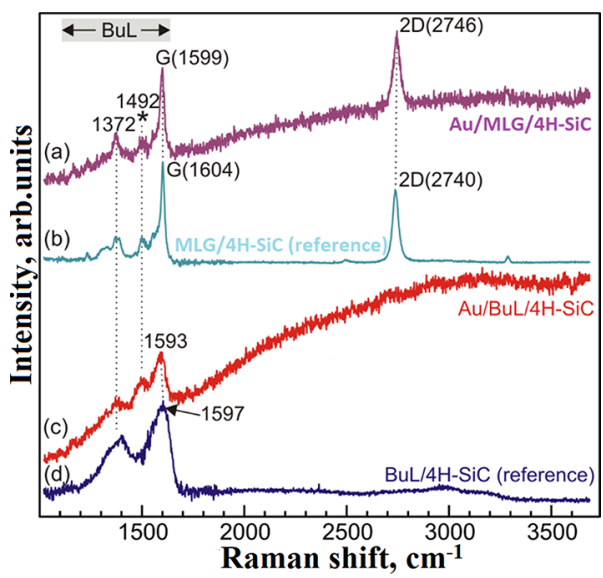

Figure 5. Typical Raman spectra of (a) Au/MLG/4H-SiC, (b) MLG/4H-SiC (reference), (c) $\mathrm{Au} / \mathrm{BuL} / 4 \mathrm{H}-\mathrm{SiC}$, and (d) the buffer layer reference $\left(\mathrm{cm}^{-1}\right)$. The reference spectra are recorded with higher excitation power $(17 \mathrm{~mW}$ vs $1 \mathrm{~mW}$ for the Au-deposited samples). In addition, the $\mathrm{Au}$ islands are the screening part of the Raman signal, which together with the lower excitation power stipulates the lower signal-to-noise ratio in (a) and (c). The vertical scaling is chosen for the convenient view only. The main features in the spectra are denoted with their peak positions $\left(\mathrm{cm}^{-1}\right)$ (for $G$ and $2 D$, the peak positions are for the spectra displayed; the statistical distribution is given in the Supporting Information). The appearance of the relatively pronounced background in the Raman spectra after $\mathrm{Au}$ deposition is ascribed to luminescence phenomena. If we consider the Raman luminescence background maximum at $3100 \mathrm{~cm}^{-1}$, with a $532 \mathrm{~nm}$ excitation wavelength, it gives a PL signal at $\sim 637 \mathrm{~nm}$, which is in very good agreement with the localized surface plasmon resonance obtained from absorbance.

buffer layer exhibits two broad phonon bands within the frequency range peaking at 1380 to $1597 \mathrm{~cm}^{-1}$, which match qualitatively the theoretically predicted vibrational density of states (vDOS) of the buffer layer on $\mathrm{SiC}^{56}$

Raman mapping confirms the formation of $\mathrm{BuL}$ within the whole investigated area, which is evidenced by the observation of the abovementioned two bands (Figure S1, Supporting Information). Since the Raman signature of the buffer layer is not related to specific discrete Raman modes (unlike the graphene case), but is instead density-of-states-like, we discuss only qualitatively the effect of $\mathrm{Au}$ on the phonon properties of the BuL. After gold deposition, the Raman spectra of the buffer layer undergo a substantial change (Figure 5 and Figure S7a, Supporting Information). More precisely, the low-frequency band is replaced by two bands peaking at about 1372 and 1492 $\mathrm{cm}^{-1}$ (the latter is denoted by an asterisk (*) in Figure 5). The new features with respect to the spectrum of pristine $\mathrm{BuL}$ are observed over the whole mapped area (see also Figure S7b,c). These changes indicate structural modifications of the $\mathrm{BuL}$ induced by interaction that entails a modification of the vibrational density of states and, consequently, of the experimental Raman spectra of the buffer layer. All measured spectra (121 in total) on the Raman map are similar, indicating a homogenous deposition of $\mathrm{Au}$ islands over the surface of the $\mathrm{BuL}$ (cf. the mapping results in Figure S7b,c, Supporting Information).

The Raman spectrum of the Au-covered graphene monolayer expressed in the $G$ and $2 D$ peaks is remarkably like that of the reference sample (apart from minor peak shifts and broadening to be discussed below), but the buffer layer contribution experiences a significant change after the $\mathrm{Au}$ deposition, if compared to the $\mathrm{BuL}$ spectrum of the MLG/4H$\mathrm{SiC}$ reference. We notice that the $\mathrm{BuL}$ contributions for the BuL sample and for the MLG/4H-SiC sample have quite different structures. However, after Au deposition, the buffer layer contributions for the samples with and without graphene have very similar structures comprising three main bands already discussed in the previous paragraph regarding the $\mathrm{BuL}$ after $\mathrm{Au}$ deposition [cf. Figure 5a,c]. The reason for this similarity is most probably due to an interaction between $\mathrm{Au}$ atoms and $\mathrm{BuL}$; in the case of the MLG/BuL/4H-SiC sample, it is due to a possible gold intercalation underneath the topmost graphene layer. To justify this assumption, we simulated eventual vertical $\mathrm{Au}$ diffusion through the hollow sites of MLG (note: an empirically measured Au atomic radius of $0.135 \mathrm{~nm}^{57}$ is very close to the geometrical graphene pore size of $0.246 \mathrm{~nm},{ }^{58}$ which indicates a nonzero probability of $\mathrm{Au}$ penetration beneath graphene). The simulation details are shown in Section S2 (Supporting Information). By analyzing Figure 6 , we noticed the presence of a high barrier $(6.84 \mathrm{eV})$ at $z=28.53 \AA$. This point of the potential energy profile can be regarded as a transition state for which we observed a pore size

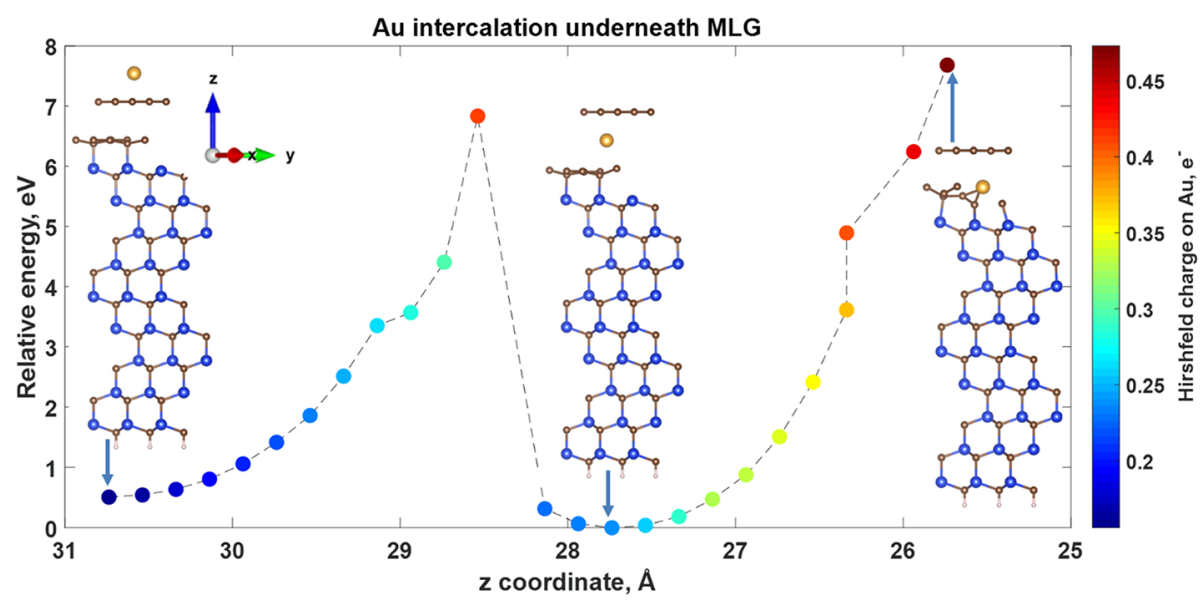

Figure 6. Free-energy profiles (color coded with Hirshfeld charge on $\mathrm{Au}$ ) corresponding to intercalation of Au underneath the topmost graphene layer in MLG/BuL/4H-SiC. The most stable adsorption site has energy of $0 \mathrm{eV}$, and less stable sites have positive energies. The optimized geometrical structures corresponding to different intercalation stages are illustrated inside the figure. 

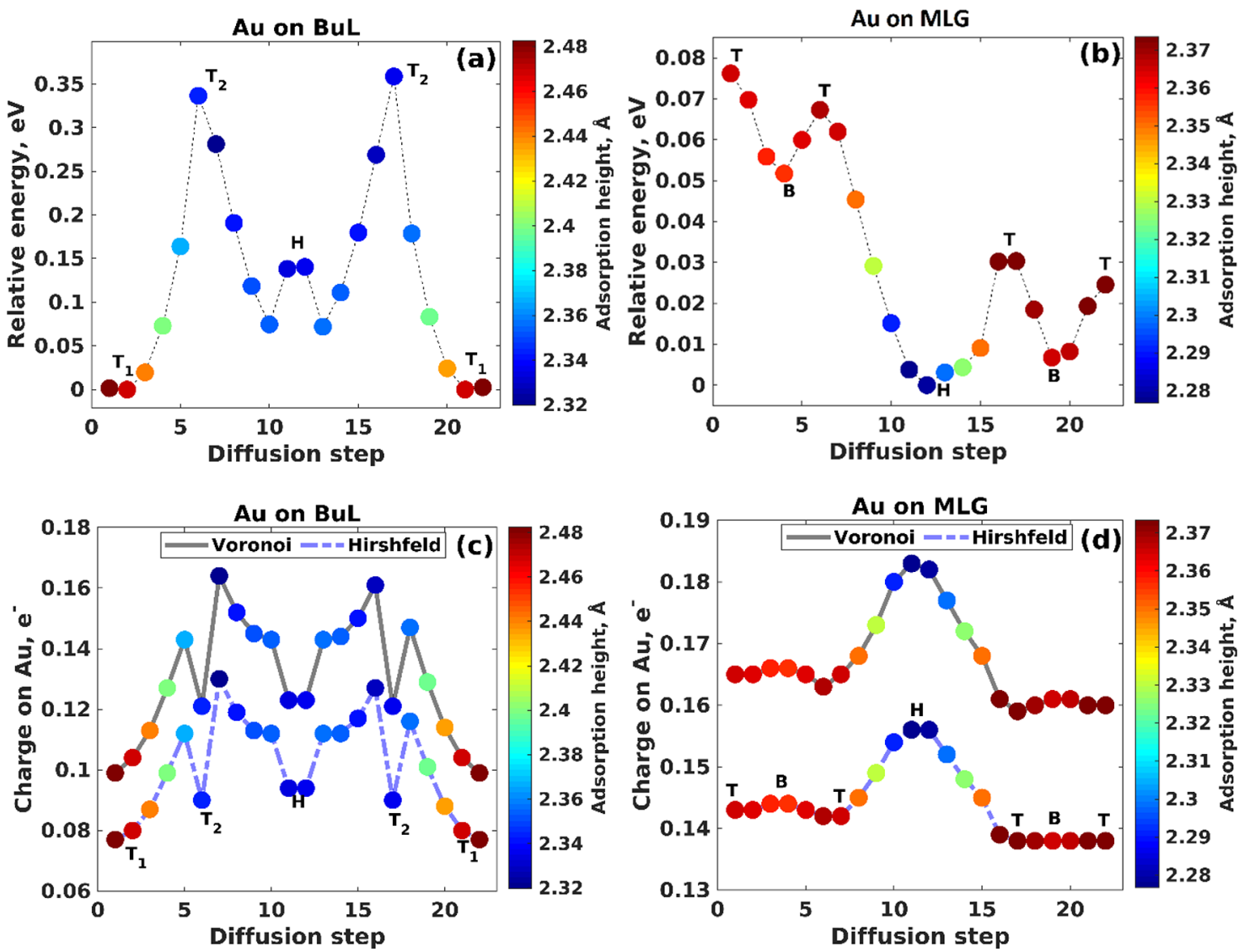

Figure 7. Free-energy profiles (color coded with Au adsorption height) corresponding to migration of Au along the diffusion path over (a) buffer layer and (b) monolayer epitaxial graphene surfaces. The most stable adsorption site has energy of $0 \mathrm{eV}$, and less stable sites have positive energies. Fluctuations of the effective charge on a Au adatom at each stage of the diffusion path for Au movement on (c) BuL and (d) MLG. The charge population analysis has been performed by using Hirshfeld and Voronoi schemes, respectively.

expansion and a final destruction of the hexagonal ring of graphene (see the Figure S8, Supporting Information). Our calculations show that the intercalation barrier is causally related to the penalty for escaping the hollow site above graphene in order to occupy the hollow site from the other side of graphene. Since the sputtered Au target atoms in our experiment have energies in the order of few electron volts with energy tails of several tens of electron volts, ${ }^{20}$ the kinetic energies of selected $\mathrm{Au}$ atoms can be compatible with the energies needed for $\mathrm{Au}$ intercalation. Thus, some fraction of $\mathrm{Au}$ atoms can be accommodated in the space between MLG and $\mathrm{BuL}$ (the middle structure inside Figure 6), thereby influencing the phonon dispersion of $\mathrm{BuL}$ in the same manner as in the cases of the graphene-free sample.

Another important argument based on our computations is that the MLG/BuL/4H-SiC system with intercalated $\mathrm{Au}$ atoms has more negative Gibbs free energy with respect to the MLG/ $\mathrm{BuL} / 4 \mathrm{H}-\mathrm{SiC}$ system with just the adsorbed $\mathrm{Au}$ atom. This implies that intercalation of $\mathrm{Au}$ atoms with high enough energy may be energetically a more favorable process than the Au adsorption. Such an advantage could provide additional driving force for $\mathrm{Au}$ intercalation. Moreover, the energy barriers for $\mathrm{Au}$ penetration beneath the buffer layer are higher than for MLG (Figure 6). Under these conditions, one could expect the growth of $2 \mathrm{D} \mathrm{Au}$ in the confined space between MLG and BuL.

It should be also noticed that the MLG after Au deposition preserves its characteristic Raman modes, which appear slightly shifted (downshift to $\sim 1597.75 \pm 1.52 \mathrm{~cm}^{-1}$ for the $G$ peak and upshift to $2742.22 \pm 4.72 \mathrm{~cm}^{-1}$ for the $2 D$ peak) relative to those of pristine MLG [Figure 5a,b, see also Figure S9a, Supporting Information]. More significantly, no defect-related Raman peaks were observed, this pointing to the robustness of epitaxial graphene upon Au deposition by sputtering. Further information can be acquired by inspecting the $2 D$ vs $G$ peak position dependence (Figure S9b). For Au-covered MLG, the points scatter primarily along a line (called the strain line) with a slope of 2.4, partly overlapping the same line for the pristine MLG sample. However, the data points shift to the left with respect to the strain line and the main group of data points for pristine MLG, which can be interpreted because of reduction of the electron density in MLG due to a screening effect. ${ }^{59}$ The nature of such a screening effect could be related to Au intercalation that weakens MLG-substrate interaction, decreasing the charge transfer from the buffer layer. Furthermore, the color-coded Raman maps (Figure S9c-e, Supporting Information) exhibit inhomogeneous broadening of $G$ and $2 D$ peaks for the MLG after Au deposition, which can be attributed to spatial charge and/or strain fluctuations at the interface.

In overall, the Raman data indicate that there is a significantly weaker interaction between $\mathrm{Au}$ and MLG compared to that between $\mathrm{Au}$ and $\mathrm{BuL}$. This aspect is further explored in sections 3.3 and 3.4, where the correlation between deposit-substrate interaction strength and the experimentally 
observed layer morphology is established using DFT calculations.

3.3. Potential Energy Landscapes for Gold Migration on Graphenized Surfaces of SiC. To quantitatively explain the experimental observations and to understand the kinetics of $\mathrm{Au}$ on $\mathrm{BuL}$ and MLG, we investigated the relative potential energy profiles (i.e., landscapes) for in-plane migration of a single $\mathrm{Au}$ adatom above both considered surfaces along predefined migration paths with a $0.3 \AA$ translation step (see Figure S5, Supporting Information). A Au adatom is allowed to relax only along the $z$ Cartesian vector, while the atomic movements along the $x$ and $y$ directions were constrained. The relative energy profiles color coded by Au adsorption height for the $\mathrm{Au}$ on BuL and MLG are plotted in Figure 7a,b, while the corresponding optimized structures related to different migration steps are shown in Figures S10 and S11 (Supporting Information), respectively.

We start by describing the Au migration on BuL. According to the data in Figure $7 \mathrm{a}$, there is a significant energy difference of $350 \mathrm{meV}$ between the minimum-energy adsorption site $\left(T_{1}\right)$ and maximum-energy adsorption on-top site position $T_{2}$ (above $\mathrm{sp}^{3}$-bonded carbon). The center of the distorted hexagonal ring with evident protrusions of $\mathrm{sp}^{2}$-bonded $\mathrm{C}$ atoms is also an energetically unfavorable adsorption position. Thus, on the $\mathrm{BuL}, \mathrm{Au}$ atoms prefer to occupy the top site position above the protruded $\mathrm{sp}^{2}$-bonded carbon atom of the $\mathrm{BuL}\left(T_{1}\right.$ position). The situation is different for the MLG surface (Figure $7 \mathrm{~b}$ ) on which the $\mathrm{Au}$ adatom favors the hollow site position $H$ (center of the hexagonal ring). The analysis of the relative energy profile for the $\mathrm{Au}$ atom on the MLG surface suggests negligibly small diffusion barriers (energy differences of $10-20 \mathrm{meV}$ ) among the adsorption sites (Figure $7 \mathrm{~b}$ ). Molecular dynamics calculations performed at room temperature for both considered systems additionally corroborate the abovementioned findings (see Section S3, Supplementary Information). Indeed, root mean square displacement (RMSD) in the case of Au/MLG is steadily increasing with time, which can be interpreted as a consequence of the weak interaction with graphene (Figure S12, Supporting Information). Meanwhile, the RMSD curve for $\mathrm{Au} / \mathrm{BuL}$ contains pronounced maximum and minimum, suggesting that the gold atom fluctuates around a stable adsorption site.

In addition to diffusion, we also studied the Au-positiondependent charge transfer on both surfaces (Figure $7 c, d$ ), which can be related to Au sharing electrons between different number of carbon atoms at each diffusion stage. More specifically, the largest charge transfer occurs at the hollow site for Au on MLG, which is energetically the most favorable adsorption site. Meanwhile, in the case of Au adsorption on the preferred $T_{1}$ site at the BuL surface, the charge transfer is minimal. According to Voronoi and Hirshfeld population analyses, the $\mathrm{Au}$ adatom donates electrons to both $\mathrm{BuL}$ and MLG, which is evidenced by the positive charge of $\mathrm{Au}$ at all positions along the diffusion path. The obtained results suggest that it is easier for electrons to be transferred from the $\mathrm{Au}$ adatom to MLG than to BuL at each diffusion stage (see also Figure S13, Supporting Information). Since a carbon-rich buffer layer has partial $\mathrm{sp}^{3}$ hybridization, some of the carbon atoms cannot accept electrons due to the complete saturation of the covalent bonding, causing an overall reduced charge transfer from Au.

From the comparison of the relative energy profiles for gold on BuL and MLG, it can be determined that the probability for trapping gold adsorbates by the $\mathrm{BuL}$ is much higher than that by the MLG. As a direct consequence, the large energetic difference between $T_{1}$ and $T_{2}$ sites for the BuL surface will decrease the diffusion length of the $\mathrm{Au}$ adatom, thereby causing the formation of a larger number of early-stage nuclei compared to the MLG surface. On the contrary, the small energetic difference in the case of MLG results in an increased diffusion length of $\mathrm{Au}$, which yields smaller and larger $\mathrm{Au}$ islands. As these islands attain relatively large size (compared to their counterparts on BuL); their shape becomes heavily dependent on the atomic diffusion at the island edges. ${ }^{60}$ Considering that $\mathrm{Au}$ is deposited at a pressure-distance product of $82.5 \mathrm{~Pa}-\mathrm{cm}$, at which gas-phase collisions are frequent and vapor flux becomes largely thermalized, ${ }^{61,62}$ we attribute the dendritic shape of the Au nanostructures on MLG to limited edge diffusion that prevails during their formation and growth. ${ }^{60}$ From a fundamental point of view, the earlystage growth morphology of vapor-deposited films is governed by the atomic-scale processes, which are operating during island nucleation, growth, and coalescence. Since in weakly interacting film/substrate systems ${ }^{63,64}$ the nominal thickness of $2 \mathrm{~nm}$ is about an order of magnitude larger than the critical thickness at which island density saturates and film morphology is governed by nucleation, it is highly likely that island coalescence is the dominant structure-forming process. Hence, the results in Figure 1 indicate faster island reshaping during $\mathrm{Au}$ coalescence on $\mathrm{BuL} / \mathrm{SiC}$, while in the $\mathrm{Au} / \mathrm{MLG} /$ $\mathrm{SiC}$ system, Au cannot completely coalesce to form rounded islands and become irregularly shaped. Unexpectedly, the growth landscape of gold on MLG resembles more Au growth on graphite than on monolayer graphene. ${ }^{65}$ Indeed, as was shown by $\mathrm{Liu}$ et al., ${ }^{65} 5 \AA \mathrm{Au}$ on graphene $/ \mathrm{SiO}_{2} / \mathrm{Si}$ is composed of many well-dispersed, compact Au nanoparticles, while using a graphite substrate led to a formation of ramified islands. Due to the reduced adatom mobility on graphene/ $\mathrm{SiO}_{2} / \mathrm{Si}$ in comparison to graphite, much smaller $\mathrm{Au}$ island density is expected on graphene than on graphite. ${ }^{65}$ The discrepancy between our experimental data and literature strongly suggests that the $\mathrm{SiC}$ substrate significantly weakens the interaction between the graphene layer and gold nanostructures, thereby favoring smaller density and larger size of $\mathrm{Au}$ atoms.

3.4. Adsorption and Nucleation of $\mathrm{Au}_{n=1-7}$ Clusters on BuL and MLG Surfaces. The details of adsorption and nucleation energy calculations are set in Sections S4-S6 (Supporting Information). The summary for single $\mathrm{Au}$ adatoms is that $\mathrm{Au}$ preferentially occupies the top site of $\mathrm{BuL}$ above the $\mathrm{sp}^{2}$-bonded carbon atom (Figure S14a, Supporting Information) and the hollow site of MLG (Figure S15a, Supporting Information), respectively. $\mathrm{Au}$ on $\mathrm{BuL}$ has adsorption energy of $0.820 \mathrm{eV}$, being larger than that of the $\mathrm{Au} / \mathrm{MLG}$ system $(0.144 \mathrm{eV})$. Such a distinct difference provides clear evidence for chemisorption of $\mathrm{Au}$ on the $\mathrm{BuL}$ surface and weak Au physisorption, mainly governed by van der Waals forces, on the MLG surface. ${ }^{66}$ For multiple gold atoms, an important finding is that the Au clusters on BuL are more stable than those on MLG, which is confirmed by the larger $E_{\text {ads }}^{2}$ (note that this energy includes two components: metal-template interaction and cohesive energies) values for all considered cases.

Finally, as can be seen from Table S5 (Supporting Information), the nucleation energy of $\mathrm{Au}$ on $\mathrm{BuL}$ and MLG is negative for all considered clusters. The obtained results 
clearly demonstrate that the metal clustering is preferred on the monolayer epitaxial graphene compared to the buffer layer, which is supported by the more negative values of $E_{\text {nuc }}$. From the experimental point of view, such a difference will correlate with a difference in the nucleation rates. To be more specific, the existing $\mathrm{Au}$ clusters on MLG can capture easier newly incoming $\mathrm{Au}$ atoms to form larger clusters than those on BuL. To sum up, $\mathrm{Au}$ on $\mathrm{BuL}$ has much larger adsorption energy, higher energetic difference between minimum-energy and maximum-energy adsorption sites, and lower diffusivity than $\mathrm{Au}$ on MLG. As a direct consequence of that, $\mathrm{Au}$ atoms or small clusters on $\mathrm{BuL}$ prefer to stick to the most favorable adsorption sites, thereby resulting in the growth of a high density of $\mathrm{Au}$ islands. Furthermore, the restricted $\mathrm{Au}$ adsorption on top site positions (above the $\mathrm{C}$ atom that is covalently bonded to $\mathrm{Si}$ ) of $\mathrm{BuL}$ prohibits, to some extent, the $\mathrm{Au}$ adatoms' diffusion on the BuL, thereby also causing the formation of a large amount of nucleation centers followed by self-assembling of a discontinuous island-like film (see Figure la,b). In contrary, Au species adsorbed on MLG are mobile and have enough time for self-assembling in large clusters.

\section{CONCLUSIONS}

Combining experimental investigations and density functional theory calculations, we explored surface kinetics of magnetronsputtered gold on carbon-rich buffer layer and monolayer epitaxial graphene on $4 \mathrm{H}-\mathrm{SiC}$. We demonstrated the selectivity of Au growth morphology on buffer layer with MLG patches. The difference in surface energetics of both graphenized surfaces is anticipated to play a major role in determining not only growth evolution of gold nanostructures but also in controlling the wettability and permeability of the topmost graphene layer. Gold on buffer layer exhibits dispersed nanoscale morphology of rounded islands, while a dendriticshaped landscape appeared on MLG. Monolayer graphene is suggested to be nontransparent to the interaction between the underlying buffer layer and gold nanostructures; thus, the formation of large ramified and fractal-like $\mathrm{Au}$ islands on $\mathrm{MLG} / \mathrm{BuL} / 4 \mathrm{H}-\mathrm{SiC}$ is mainly driven by weak van der Waals interaction between $\mathrm{Au}$ and graphene. These are prerequisites of easy self-organization of gold and prevention of defect generation. As was confirmed by the DFT results, the morphological distinctiveness of gold on both substrates is intrinsically linked to the adsorption capacity of the surface and substrate-influenced $\mathrm{Au}$ diffusivity. The unique corrugated topology of the buffer layer related to the mixed $\mathrm{sp}^{2}-\mathrm{sp}^{3}$ chemical bonding is identified to be decisive for the high $\mathrm{Au}$ adsorption and limited diffusivity. The current results shed light on the atomistic level interplay between gold clusters and graphenized surfaces of $4 \mathrm{H}-\mathrm{SiC}$ and can be exploited to conceptualize novel applications in catalysis, sensorics, and spintronics. We found that during the early nucleation stages, gold atoms (from 2 to 7 ) supported by nanostructured surfaces of $\mathrm{SiC}$ choose planar configuration rather than the threedimensional one. Furthermore, we identified an odd-numbered $\mathrm{Au}_{3}$ cluster as a minimal structural unit, acting as a growth nucleus. Raman results complemented by $a b$ initio predictions provide reasoned arguments in favor of in situ gold intercalation beneath monolayer graphene, thereby opening a way for realization of, e.g., spatially confined $2 \mathrm{D}$ metal growth. Our finding also suggests that coexisting buffer layermonolayer graphene regions providing unique adsorption capacity with respect to gold can be beneficial for designing novel robust artificial materials with patterned nanoscale wettability and selective electronic performance. The strong affinity of transition metals on buffer layer can be used as a chemical sensor that can detect catalytic amount of organic and inorganic gases, such as benzene and $\mathrm{NO}_{x}$. Furthermore, the observation of localized surface plasmon resonance in golddecorated buffer layer could open the possibility for optical sensing of biomolecules. Further studies may provide a new platform for catalytic processes and biodetection.

\section{ASSOCIATED CONTENT}

\section{(I) Supporting Information}

The Supporting Information is available free of charge at https://pubs.acs.org/doi/10.1021/acsanm.0c02867.

Raman data for bare buffer layer; Raman data for bare monolayer epitaxial graphene; reflectance map of bare monolayer epitaxial graphene; the $2 \times 2$ supercell of buffer layer and monolayer epitaxial graphene; the migration path; the $4 \times 4$ supercell of buffer layer and monolayer epitaxial graphene; molecular dynamics results; Raman data for Au-decorated buffer layer; intercalation of gold; Raman data for Au-decorated monolayer epitaxial graphene; optimized structures of 3D Au islands; optimized structures of 2D Au islands; adsorption of gold clusters; and parameters describing gold-template interaction (PDF)

\section{AUTHOR INFORMATION}

\section{Corresponding Author}

Ivan Shtepliuk - Semiconductor Materials Division, Department of Physics, Chemistry and Biology-IFM, Linköping University, Linköping S-58183, Sweden; ○ orcid.org/0000-0002-8685-3332;

Email: ivan.sthepliuk@liu.se

\section{Authors}

Ivan G. Ivanov - Semiconductor Materials Division, Department of Physics, Chemistry and Biology-IFM, Linköping University, Linköping S-58183, Sweden

Nikolaos Pliatsikas - Nanoscale Engineering Division, Department of Physics, Chemistry and Biology-IFM, Linköping University, Linköping S-58183, Sweden; (1) orcid.org/0000-0002-5098-6950

Tihomir Iakimov - Semiconductor Materials Division, Department of Physics, Chemistry and Biology-IFM, Linköping University, Linköping S-58183, Sweden; (1) orcid.org/0000-0003-2256-4977

Samuel Lara-Avila - Quantum Device Physics Laboratory, Department of Microtechnology and Nanoscience, Chalmers University of Technology, Göteborg S-41296, Sweden; (1) orcid.org/0000-0002-8331-718X

Kyung Ho Kim - Quantum Device Physics Laboratory, Department of Microtechnology and Nanoscience, Chalmers University of Technology, Göteborg S-41296, Sweden; (1) orcid.org/0000-0002-2029-729X

Nabiha Ben Sedrine - Department of Physics and I3N, Institute for Nanostructures, Nanomodelling and Nanofabrication, University of Aveiro, Aveiro 3810-193, Portugal; 10 orcid.org/0000-0002-2255-3453

Sergey E. Kubatkin - Quantum Device Physics Laboratory, Department of Microtechnology and Nanoscience, Chalmers 
University of Technology, Göteborg S-41296, Sweden; (1) orcid.org/0000-0001-8551-9247

Kostas Sarakinos - Nanoscale Engineering Division, Department of Physics, Chemistry and Biology-IFM, Linköping University, Linköping S-58183, Sweden; (1) orcid.org/0000-0003-2864-9509

Rositsa Yakimova - Semiconductor Materials Division, Department of Physics, Chemistry and Biology-IFM, Linköping University, Linköping S-58183, Sweden; (1) orcid.org/0000-0003-4237-2702

Complete contact information is available at: https://pubs.acs.org/10.1021/acsanm.0c02867

\section{Author Contributions}

I.S. performed all theoretical calculations and prepared the original draft of the paper. K.S. and N.P. were responsible for magnetron sputtering of $\mathrm{Au}$, morphology characterization, and contribution to writing. I.G.I conducted reflectance mapping analysis and the Raman study. T.I. fabricated the buffer layer and epitaxial graphene samples. K.H.K. and S.L.-A. deposited gold layers through thermal evaporation and performed the AFM study. N.B.S. performed absorbance measurements. The original idea of the current research was proposed by R.Y. and S.K. The paper was written under the supervision of R.Y. All authors have given approval to the final version of the paper.

\section{Notes}

The authors declare no competing financial interest.

\section{ACKNOWLEDGMENTS}

I.S. and K.S. acknowledge the support from Ångpanneföreningens Forskningsstiftelse (grants 16-541 and 19-137). This work was developed within the scope of the project I3N, UIDB/50025/2020, \& UIDP/50025/2020 and financed by national funds through the Fundação para a Ciência e a Tecnologia/Ministério da Educação e Ciências (FCT/MEC). K.S. acknowledges financial support from Linköpings Universitet ("LiU Career Contract, Dnr-LiU-2015-01510, 20152020”). Financial support by Stiftelsen för Strategisk Forskning via grant RMA 15-0024 is greatly acknowledged. K.S. and N.P. acknowledge financial support from Stiftelsen Olle Engkvist Byggmästare (contract SOEB 190-312). R.Y., I.G.I., and K.S. received funding from Vetenskapsrådet (grants 2018-04962, 2016-05362, and 2015-04630). K.S and N. P. have been (partially) supported by the Wenner-Gren Stiftelserna (contracts UPD2018-0071 and UPD2019-0007).

\section{REFERENCES}

(1) He, H.; Lara-Avila, S.; Kim, K. H.; Fletcher, N.; Rozhko, S.; Bergsten, T.; Eklund, G.; Cedergren, K.; Yakimova, R.; Park, Y. W.; Tzalenchuk, A.; Kubatkin, S. Polymer-encapsulated molecular doped epigraphene for quantum resistance metrology. Metrologia 2019, 56, No. 045004.

(2) Tzalenchuk, A.; Lara-Avila, S.; Kalaboukhov, A.; Paolillo, S.; Syväjärvi, M.; Yakimova, R.; Kazakova, O.; Janssen, T. J. B. M.; Fal'ko, V.; Kubatkin, S. Towards a quantum resistance standard based on epitaxial graphene. Nature Nanotech. 2010, 5, 186.

(3) Janssen, T. J. B. M.; Rozhko, S.; Antonov, I.; Tzalenchuk, A.; Williams, J. M.; Melhem, Z.; He, H.; Lara-Avila, S.; Kubatkin, S.; Yakimova, R. Operation of graphene quantum Hall resistance standard in a cryogen-free table-top system. $2 D$ Mater. 2015, 2, 035015.

(4) Hertel, S.; Waldmann, D.; Jobst, J.; Albert, A.; Albrecht, M.; Reshanov, S.; Schöner, A.; Krieger, M.; Weberm, H. B. Tailoring the graphene/silicon carbide interface for monolithic wafer-scale electronics. Nat. Commun. 2012, 3, 957.

(5) Lin, Y.-M.; Dimitrakopoulos, C.; Jenkins, K. A.; Farmer, D. B.; Chiu, H.-Y.; Grill, A.; Avouris, P. 100-GHz transistors from waferscale epitaxial graphene. Science 2010, 327, 662.

(6) He, H.; Kim, K. H.; Danilov, A.; Montemurro, D.; Yu, L.; Park, Y. W.; Lombardi, F.; Bauch, T.; Moth-Poulsen, K.; Iakimov, T.; Yakimova, R.; Malmberg, P.; Müller, C.; Kubatkin, S.; Lara-Avila, S. Uniform doping of graphene close to the Dirac point by polymerassisted assembly of molecular dopants. Nat. Commun. 2018, 9, 3956. (7) Santangelo, M. F.; Shtepliuk, I.; Filippini, D.; Ivanov, I. G.; Yakimova, R.; Eriksson, J. Real-time sensing of lead with epitaxial graphene-integrated microfluidic devices. Sens. Actuators, B Chem. 2019, 288, 425.

(8) Melios, C.; Panchal, V.; Edmonds, K.; Lartsev, A.; Yakimova, R.; Kazakova, O. Detection of Ultralow Concentration $\mathrm{NO}_{2}$ in Complex Environment Using Epitaxial Graphene Sensors. ACS Sens. 2018, 3, 1666.

(9) Melios, C.; Winters, M.; Strupiński, W.; Panchal, V.; Giusca, C. E.; Jayawardena, K. D. G. I.; Rorsman, N.; Silva, S. R. P.; Kazakova, O. Tuning epitaxial graphene sensitivity to water by hydrogen intercalation. Nanoscale 2017, 9, 3440.

(10) Lara-Avila, S.; Danilov, A.; Golubev, D.; He, H.; Kim, K. H.; Yakimova, R.; Lombardi, F.; Bauch, T.; Cherednichenko, S.; Kubatkin, S. Towards quantum-limited coherent detection of terahertz waves in charge-neutral graphene. Nat. Astron. 2019, 3, 983-988.

(11) Shtepliuk, I.; Khranovskyy, V.; Yakimova, R. Combining graphene with silicon carbide: synthesis and properties - a review. Semicond. Sci. Technol. 2016, 31, 113004.

(12) Druga, T.; Wenderoth, M.; Lüpke, F.; Ulbrich, R. G. Graphenemetal contact resistivity on semi-insulating $6 \mathrm{H}-\mathrm{SiC}(0001)$ measured with Kelvin probe force microscopy. Appl. Phys. Lett. 2013, 103, No. 051601.

(13) Daniels, K. M.; Obe, A.; Daas, B. K.; Weidner, J.; Williams, C.; Sudarshan, T. S.; Chandrashekhar, M. V. S. Metal Catalyzed Electrochemical Synthesis of Hydrocarbons from Epitaxial Graphene. J. Electrochem. Soc. 2016, 163, E130.

(14) Hossain, M. Z.; Shimizu, N. Covalent Immobilization of Gold Nanoparticles on Graphene. J. Phys. Chem. C 2019, 123, 3512.

(15) Chadhari, S.; Graves, A. R.; Cain, M. V.; Stinespring, C. D. Graphene-based composite sensors for energy applications. Proc. SPIE Int. Soc. Opt. Eng. 2016, 9836, 98360G.

(16) Eriksson, J.; Puglisi, D.; Kang, Y. H.; Yakimova, R.; Lloyd Spetz, A. Adjusting the electronic properties and gas reactivity of epitaxial graphene by thin surface metallization. Phys. B 2014, 439, 105.

(17) Matsumura, H.; Yanagiya, S.-I.; Nagase, M.; Kishikawa, H.; Goto, N. Microscopic Raman study of graphene on $4 \mathrm{H}-\mathrm{SiC}$ twodimensionally enhanced by surface roughness and gold nanoparticles. Jpn. J. Appl. Phys. 2016, 55, No. 06GL05.

(18) Niu, J.; Truong, V. G.; Huang, H.; Tripathy, S.; Qiu, C.; Wee, A. T. S.; Yu, T.; Yang, H. Study of electromagnetic enhancement for surface enhanced Raman spectroscopy of SiC graphene. Appl. Phys. Lett. 2012, 100, 191601.

(19) Shtepliuk, I.; Ivanov, I. G.; Pliatsikas, N.; Iakimov, T.; Jamnig, A.; Sarakinos, K.; Yakimova, R. Probing the uniformity of silver-doped epitaxial graphene by micro-Raman mapping. Phys. B 2020, 580, 411751 .

(20) Shtepliuk, I.; Ivanov, I. G.; Pliatsikas, N.; Sedrine, N. B.; Andersson, O.; Iakimov, T.; Jamnig, A.; Sarakinos, K.; Yakimova, R. Interplay between thin silver films and epitaxial graphene. Surf. Coat. Technol. 2020, 381, 125200.

(21) Caccia, M.; Giuranno, D.; Molina-Jorda, J. M.; Moral, M.; Nowak, R.; Ricci, E.; Sobczak, N.; Narciso, J.; Fernández Sanz, J. Graphene Translucency and Interfacial Interactions in the Gold/ Graphene/SiC System. J. Phys. Chem. Lett. 2018, 9, 3850.

(22) DeJarld, M.; Campbell, P. M.; Friedman, A. L.; Currie, M.; Myers-Ward, R. L.; Boyd, A. K.; Rosenberg, S. G.; Pavunny, S. P.; Daniels, K. M.; Gaskill, D. K. Surface potential and thin film quality of 
low work function metals on epitaxial graphene. Sci. Rep. 2018, 8, 16487.

(23) Briggs, N.; Gebeyehu, Z. M.; Vera, A.; Zhao, T.; Wang, K.; De La Fuente Duran, A.; Bersch, B.; Bowen, T.; Knappenberger, K. L.; Robinson, J. A. Epitaxial graphene/silicon carbide intercalation: a minireview on graphene modulation and unique $2 \mathrm{D}$ materials. Nanoscale 2019, 11, 15440.

(24) Kim, K. H.; He, H.; Rodner, M.; Yakimova, R.; Larsson, K.; Piantek, M.; Serrate, D.; Zakharov, A.; Kubatkin, S.; Eriksson, J.; LaraAvila, S. Chemical Sensing with Atomically Thin Platinum Templated by a 2D Insulator. Adv. Mater. Interfaces 2020, 7, 1902104.

(25) Kim, K. H.; He, H.; Struzzi, C.; Zakharov, A.; Giusca, C. E.; Tzalenchuk, A.; Park, Y. W.; Yakimova, R.; Kubatkin, S.; Lara-Avila, S. Ambipolar charge transport in quasi-free-standing monolayer graphene on SiC obtained by gold intercalation. Phys. Rev. B 2020, 102, 165403.

(26) Bayani, A.; Larsson, K. The morphology of an intercalated Au layer with its effect on the Dirac point of graphene. Sci. Rep. 2020, 10, 1042

(27) Narayanan Nair, M.; Cranney, M.; Jiang, T.; Hajjar-Garreau, S.; Aubel, D.; Vonau, F.; Florentin, A.; Denys, E.; Bocquet, M.-L.; Simon, L. Noble-metal intercalation process leading to a protected adatom in a graphene hollow site. Phys. Rev. B 2016, 94, No. 075427.

(28) Marchenko, D.; Varykhalov, A.; Sánchez-Barriga, J.; Seyller, T.; Rader, O. Rashba splitting of $100 \mathrm{meV}$ in Au-intercalated graphene on SiC. Appl. Phys. Lett. 2016, 108, 172405.

(29) Forti, S.; Link, S.; Stöhr, A.; Niu, Y.; Zakharov, A. A.; Coletti, C.; Starke, U. Semiconductor to metal transition in two-dimensional gold and its van der Waals heterostack with graphene. Nat. Commun. 2020, 11, 2236.

(30) Chuang, F.-C.; Lin, W.-H.; Huang, Z.-Q.; Hsu, C.-H.; Kuo, C.C.; Ozolins, V.; Yeh, V. Electronic structures of an epitaxial graphene monolayer on $\mathrm{SiC}(0001)$ after gold intercalation: a first-principles study. Nanotechnology 2011, 22, 275704.

(31) Cheng, Y. C.; Schwingenschlögl, U. A route to strong p-doping of epitaxial graphene on SiC. Appl. Phys. Lett. 2010, 97, 193304.

(32) Premlal, B.; Cranney, M.; Vonau, F.; Aubel, D.; Casterman, D.; De Souza, M. M.; Simon, L. Surface intercalation of gold underneath a graphene monolayer on $\mathrm{SiC}(0001)$ studied by scanning tunneling microscopy and spectroscopy. Appl. Phys. Lett. 2009, 94, 263115.

(33) Pillai, P. B.; DeSouza, M.; Narula, R.; Reich, S.; Wong, L. Y.; Batten, T.; Pokorny, J. Decoupling of epitaxial graphene via gold intercalation probed by dispersive Raman spectroscopy. J. Appl. Phys. 2015, 117, 183103.

(34) Nair, M. N.; Cranney, M.; Vonau, F.; Aubel, D.; Le Fèvre, P.; Tejeda, A.; Bertran, F.; Taleb-Ibrahimi, A.; Simon, L. High van Hove singularity extension and Fermi velocity increase in epitaxial graphene functionalized by intercalated gold clusters. Phys. Rev. B 2012, 85, 245421.

(35) Gierz, I.; Suzuki, T.; Weitz, R. T.; Lee, D. S.; Krauss, B.; Riedl, C.; Starke, U.; Höchst, H.; Smet, J. H.; Ast, C. R.; Kern, K. Electronic decoupling of an epitaxial graphene monolayer by gold intercalation. Phys. Rev. B 2010, 81, 235408.

(36) Yakimova, R.; Iakimov, T.; Syväjärvi, M. Process for Growth of Graphene. U.S. Patent US9,150,417B2, 2015.

(37) Shtepliuk, I.; Ivanov, I. G.; Iakimov, T.; Yakimova, R.; Kakanakova-Georgieva, A.; Fiorenza, P.; Giannazzo, F. Raman probing of hydrogen-intercalated graphene on Si-face $4 \mathrm{H}-\mathrm{SiC}$. Mater. Sci. Semicond. Process. 2019, 96, 145-152.

(38) Ni, Z. H.; Chen, W.; Fan, X. F.; Kuo, J. L.; Yu, T.; Wee, A. T. S.; Shen, Z. X. Raman spectroscopy of epitaxial graphene on a SiC substrate. Phys. Rev. B 2008, 77, 115416.

(39) Jamnig, A.; Pliatsikas, N.; Konpan, M.; Lu, J.; Kehagias, T.; Kotanidis, A. N.; Kalfagiannis, N.; Bellas, D. V.; Lidorikis, E.; Kovac, J.; Abadias, G.; Petrov, I.; Greene, J. E.; Sarakinos, K. 3D-to-2D morphology manipulation of sputter-deposited nanoscale silver films on weakly-interacting substrates via selective nitrogen deployment for multifunctional metal contacts. ACS Appl. Nano Mater. 2020, 3, $4728-4738$.
(40) Mattausch, A.; Pankratov, O. Ab Initio Study of Graphene on SiC. Phys. Rev. Lett. 2007, 99, No. 076802.

(41) Jayasekera, T.; Kong, B. D.; Kim, K. W.; Buongiorno Nardelli, M. Band Engineering and Magnetic Doping of Epitaxial Graphene on SiC (0001). Phys. Rev. Lett. 2010, 104, 146801.

(42) Kürpick, U.; Kara, A.; Rahman, T. S. Role of Lattice Vibrations in Adatom Diffusion. Phys. Rev. Lett. 1997, 78, 1086.

(43) Boisvert, G.; Mousseau, N.; Lewis, L. J. Comment on "Role of Lattice Vibrations in Adatom Diffusion”. Phys. Rev. Lett. 1998, 80, 203.

(44) Sangiovanni, D. G.; Mei, A. B.; Edström, D.; Hultman, L.; Chirita, V.; Petrov, I.; Greene, J. E. Effects of surface vibrations on interlayer mass transport: $\mathrm{Ab}$ initio molecular dynamics investigation of $\mathrm{Ti}$ adatom descent pathways and rates from $\mathrm{TiN} / \mathrm{TiN}(001)$ islands. Phys. Rev. B 2018, 97, No. 035406.

(45) Smirnova, D.; Starikov, S.; Leines, G. D.; Liang, Y.; Wang, N.; Popov, M. N.; Abrikosov, I. A.; Sangiovanni, D. G.; Drautz, R.; Mrovec, M. Atomistic description of self-diffusion in molybdenum: A comparative theoretical study of non-Arrhenius behavior. Phys. Rev. Mater. 2020, 4, No. 013605.

(46) Habenicht, B. F.; Teng, D.; Semidey-Flecha, L.; Sholl, D. S.; $\mathrm{Xu}, \mathrm{Y}$. Adsorption and Diffusion of $4 \mathrm{~d}$ and $5 \mathrm{~d}$ Transition Metal Adatoms on Graphene $/ \mathrm{Ru}(0001)$ and the Implications for Cluster Nucleation. Top. Catal. 2014, 57, 69.

(47) Soler, J. M.; Artacho, E.; Gale, J. D.; García, A.; Junquera, J.; Ordejón, P.; Sánchez-Portal, D. The SIESTA method for ab initio order-N materials simulation. J. Phys.: Condens. Matter 2002, 14, 2745.

(48) Berland, K.; Hyldgaard, P. Exchange functional that tests the robustness of the plasmon description of the van der Waals density functional. Phys. Rev. B 2014, 89, No. 035412.

(49) Pseudopotentials for SIESTA; https://departments.icmab.es/ leem/siesta/Pseudopotentials/.

(50) Schimka, L.; Harl, J.; Stroppa, A.; Grüneis, A.; Marsman, M.; Mittendorfer, F.; Kresse, G. Accurate surface and adsorption energies from many-body perturbation theory. Nat. Rev. Mater. 2010, 9, 741744.

(51) van Engers, C. D.; Cousens, N. E. A.; Babenko, V.; Britton, J.; Zappone, B.; Grobert, N.; Perkin, S. Direct Measurement of the Surface Energy of Graphene. Nano Lett. 2017, 17, 3815.

(52) Alaskar, Y.; Arafin, S.; Wickramaratne, D.; Zurbuchen, M. A.; He, L.; McKay, J.; Lin, Q.; Goorsky, M. S.; Lake, R. K.; Wang, K. L. Towards van der Waals Epitaxial Growth of GaAs on Si using a Graphene Buffer Layer. Adv. Funct. Mater. 2014, 24, 6629.

(53) Sun, L.; Chen, X.; Yu, W.; Sun, H.; Zhao, X.; Xu, X.; Yu, F.; Liu, Y. The effect of the surface energy and structure of the SiC substrate on epitaxial graphene growth. RSC Adv. 2016, 6, 100908.

(54) Ambrosetti, A.; Silvestrelli, P. L. Hidden by graphene-Towards effective screening of interface van der Waals interactions via monolayer coating. Carbon 2018, 139, 486-491.

(55) Bogoyavlenskiy, V. A.; Chernova, N. A. Diffusion-limited aggregation: a relationship between surface thermodynamics and crystal morphology. Phys. Rev. E 2000, 61, 1629-1633.

(56) Fromm, F.; Oliveira, M. H., Jr.; Molina-Sánchez, A.; Hundhausen, M.; Lopes, J. M. J.; Riechert, H.; Wirtz, L.; Seyller, T. Contribution of the buffer layer to the Raman spectrum of epitaxial graphene on $\mathrm{SiC}(0001)$. New J. Phys. 2013, 15, No. 043031.

(57) Slater, J. C. Atomic Radii in Crystals. J. Chem. Phys. 1964, 41, 3199.

(58) Berry, V. Impermeability of graphene and its applications. Carbon 2013, 62, 1.

(59) Eliseyev, I. A.; Davydov, V. Y.; Smirnov, A. N.; Nestoklon, M. O.; Dementev, P. A.; Lebedev, S. P.; Lebedev, A. A.; Bokai, K. A.; Usachov, D. Y. Raman spectroscopy estimation of the carrier concentration and the value of strain in monolayer graphene films grown on 4H-SiC. J. Phys.: Conf. Ser. 2019, 1400, No. 055037.

(60) Michely, T.; Krug, J. Islands, Mounds, and Atoms: Patterns and Processes in Crystal Growth Far from Equilibrium; Springer: Berlin, 2003. 
(61) Atiser, A.; Mráz, S.; Schneider, J. M. Pressure dependence of the $\mathrm{Al}$ ion energy distribution functions during filtered cathodic arc thin film growth in an Ar, $\mathrm{O}_{2}$ ambient. J. Phys. D: Appl. Phys. 2009, 42, No. 015202.

(62) Sarakinos, K.; Music, D.; Nahif, F.; Jiang, K.; Braun, A.; Zilkens, C.; Schneider, J. M. Ionized physical vapor deposited $\mathrm{Al}_{2} \mathrm{O}_{3}$ films: Does subplantation favor formation of $\alpha-\mathrm{Al}_{2} \mathrm{O}_{3}$ ? Phys. Status Solidi RRL 2010, 4, 154.

(63) Elofsson, V.; Lü, B.; Magnfält, D.; Münger, E. P.; Sarakinos, K. Unravelling the physical mechanisms that determine microstructural evolution of ultrathin Volmer-Weber films. J. Appl. Phys. 2014, 116, No. 044302.

(64) Lü, B.; Elofsson, V.; Münger, E. P.; Sarakinos, K. Dynamic competition between island growth and coalescence in metal-oninsulator deposition. Appl. Phys. Lett. 2014, 105, 163107.

(65) Liu, L.; Chen, Z.; Wang, L.; Polyakova, E. S.; Taniguchi, T.; Watanabe, K.; Hone, J.; Flynn, G. W.; Brus, L. E. Slow Gold Adatom Diffusion on Graphene: Effect of Silicon Dioxide and Hexagonal Boron Nitride Substrates. J. Phys. Chem. B 2013, 117, 4305-4312.

(66) Spanjaard, D.; Desonqueres, M. C. in Interactions of Atoms and Molecules with Solid Surfaces; Vol: 1 Eds: Bortolani, V.; March, N. H.; Tosi, M. Springer: New York, US 1990. , Ch. 9 